$2^{\text {ème }}$ version corrigée -9 novembre 2018

Tel-Aviv Ville Blanche: la construction d'un objet patrimonial et ses effets sur le développement de la ville.

\title{
Rozenholc Caroline
}

Centre de recherche sur l'habitat, UMR 7218 LAVUE

ENSA Paris-Val de Seine

3-15 Quai Panhard et Levassor, Paris $13^{\text {ème }}$

caroline.rozenholc@paris-valdeseine.archi.fr

\section{TUFAno Antonella}

Maacc, UMR 3495 MAP

ENSA Paris-La Villette

144 avenue de Flandre, Paris $19^{\text {ème }}$

antonella.tufano@paris-lavillette.archi.fr

\section{Résumé}

En 2003, Tel-Aviv est inscrite au patrimoine mondial de l'Unesco pour son architecture et son urbanisme modernes. Cette date est décisive pour l'histoire de la ville et pour sa mise en récit, comme pour les pratiques relativement libres de transformation des immeubles dits Bauhaus et de la surélévation de certains logements. Ces modifications sont alors interdites par les règles d'urbanisme, alors que, dans le même temps, certains bâtiments inscrits sont surélevés de plusieurs étages et de très nombreuses tours construites. L'étude de Tel-Aviv permet, de ce point de vue, de répondre à une question d'ordre général sur le classement au titre du patrimoine et sur les effets paradoxaux de la patrimonialisation en milieu urbain, dans un tissu qui se densifie et se transforme très rapidement, alors que des mesures conservatoires sont prises à son endroit.

\section{Introduction}

Capitale économique incontestable d'Israël, ville multiculturelle et globale ${ }^{1}$, Tel-Aviv est aussi le symbole d'une culture européenne renouvelée qui s'est implantée en Palestine au début du vingtième siècle. En 2003, un siècle après son édification en 1909, une partie de son centre-ville (dit la «Ville Blanche») a été inscrite par l'Unesco au Patrimoine Mondial de l'Humanité. Aujourd'hui, cet article souhaite revenir sur les discours qui ont présidé à cette décision internationale, sur certaines conséquences locales de cette inscription et sur l'usage, voire sur l'instrumentalisation, qui en est fait. À partir de cette étude de cas, cet article

\footnotetext{
${ }^{1}$ Depuis le milieu des années 2000 - avec l'entrée de la ville dans le marché international, l'accroissement des disparités, la marchandisation des services sociaux et l'afflux de travailleurs immigrés ou sans papier -, les chercheurs israéliens (N. Alfasi et T. Fenster, 2005 ; B. Kipnis, 2004 ; M. Levine, 2005 par exemple) analysent Tel-Aviv comme une ville globale.
} 
souhaite également contribuer aux débats émergeants sur les processus de patrimonialisation et leurs effets ; ces derniers pouvant être lus à la fois comme le fruit de négociations, souvent longues, et comme l'instrument d'un discours et d'intentions politiques lorsque l'on choisit de classer un site.

Pour ce faire, nous proposons une double lecture de la ville, morpho-typologique et socioculturelle, qui permettra de vérifier l'hypothèse suivante : le patrimoine est une entrée pour aborder des questions sociales, politiques et urbaines. Il en est le révélateur. Ce faisant, nous aborderons deux aspects de la question patrimoniale à Tel-Aviv :

(i) L'émergence de la question patrimoniale, la construction du discours patrimonial et la démarche d'inscription de l'Unesco ;

(ii) Les mutations induites dans la ville et tout particulièrement l'effet de densification qui touche ses secteurs « péri-patrimoniaux ».

Le croisement de ces deux aspects permettra ensuite de revisiter la notion de patrimoine dans une ville comme Tel-Aviv, en transformation constante et rapide, et qui, volontairement ou non, semble être devenue un argument pour séparer les strates identitaires et mémorielles de la ville. Si le devenir patrimoine d'un lieu, outre le fait d'une patrimonialisation institutionnelle, est le fruit d'une stratification de la mémoire et de l'incorporation des conflits qui sous-tendent la naissance d'une mémoire collective, à Tel-Aviv nous verrons que la patrimonialisation de la ville renforce, voire produit, certaines dynamiques de relégations de quartiers, de populations et donc de mémoires. L'obtention du prestigieux label de l'Unesco tend en effet à avoir un effet homogénéisant qui participe de l'oblitération de certains quartiers, pourtant historiques, du récit de la ville comme "Ville blanche » européenne et moderne (Rozenholc, 2009 et 2018) au moment où Tel-Aviv se mondialise - avec le développement d'enseignes internationales, la multiplication des tours et des interventions de «starchitects" (Charney, 2009; Gravari-Barbas et Renard-Delautre, 2015) dans un tissu jusque-là relativement bas, de 4 à 8 étages le plus souvent. Le rythme de ce développement, et son accélération depuis le milieu des années 2010, est tel que plus de 150 tours se sont ajoutées aux 230 que comptait déjà la ville en 2008 ; contre 179 à Haïfa et 50 à Jérusalem, les deux autres grandes villes du pays. On en recense ainsi aujourd'hui 385 (tours et gratte-ciel de 12 à 49 étages confondus) auxquels s'ajoutent encore 264 projets en cours ou planifiés (Rozenholc, 2018).

Document 1: La tour «Meier on Rothschild» : un projet controversé 


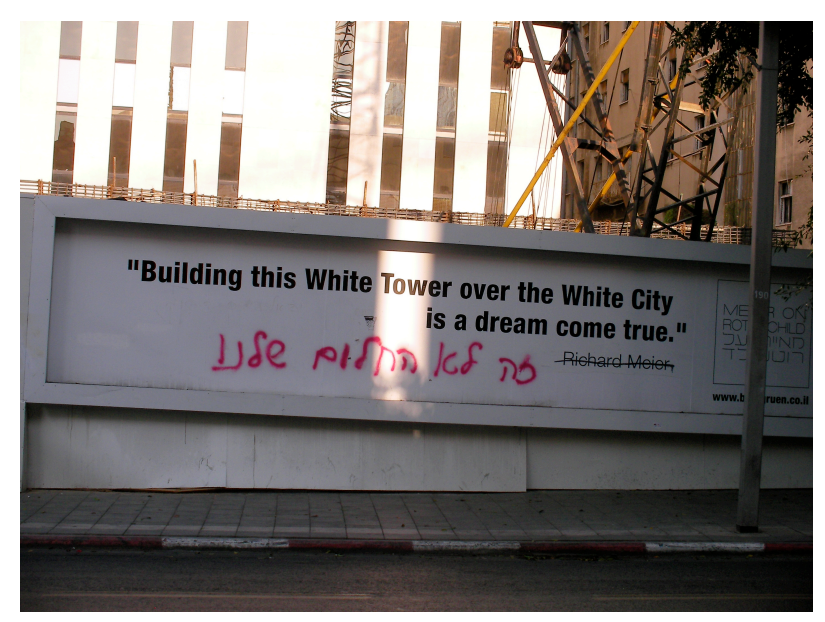

Légende: En 2008, après le musée d'Art contemporain de Barcelone et le centre Getty de Los Angeles, l'architecte américain Richard Meier, récipiendaire du prestigieux prix Pritzker, construit "par dessus » la Ville blanche l'un des plus hauts bâtiments du pays. Avec une tour de verre et d'aluminium de 158 mètres, installée au coin des rues Rothschild et Allenby, il contribue ainsi, comme d'autres «starchitects » internationaux, à transformer profondément le paysage et l'horizon de la ville. Terminé en 2014, le bâtiment compte 10 étages de plus que les 27 initialement prévus et proposait, en 2018, des appartements à la vente pour un prix minimum de 2,25 millions de dollars. Si Meier réalise son rêve de construire une "Tour Blanche au-dessus de la Ville Blanche ", dans la zone tampon du périmètre Unesco, il n'emporte pas l'assentiment de tous. En témoigne un graffiti - «ze lo ha'halom shelanou », "ça n'est pas notre rêve » - inscrit un rouge et en hébreu, à la bombe, sous le slogan en anglais de R. Meier : "Building this White Tower in the White City is a dream come true ».

Crédits : C. Rozenholc, octobre 2008.

\section{Première trace, premier tracé : la relation à la terre}

Avant d'entrer à proprement parler dans la question de la patrimonialisation de la ville, il est important de revenir sur les éléments de sa fondation. S'il est vrai que, depuis l'Antiquité, la naissance d'une ville repose sur la relation avec son contexte géomorphologique, cette relation paraît exacerbée dans le cas de Tel-Aviv. La terre et la nature jouent un rôle central dans le projet urbain sioniste (Epstein-Pliouchtch, 2012) et dans la relation fructueuse de l'homme à la Nature et à l'agriculture qu'il vise à rétablir. Cette dernière se développe d'un côté dans les collectivités rurales et, de l'autre, par la présence de jardins et d'espaces de nature maitrisée dans l'organisation du premier noyau de Tel-Aviv, Ahuzat Bait, un quartier exclusivement résidentiel de $\mathrm{Jaffa}^{2}$. En effet, si dans le récit de la fondation de la ville, les dunes et l'idée d'une ville construite sur le sable est omniprésente ${ }^{3}$, l'analyse des différents plans montre que Tel-Aviv s'insère dans un tissu de quartiers préexistants dont le tracé

\footnotetext{
${ }^{2}$ La création de Tel-Aviv est le plus souvent datée de la construction du quartier Ahuzat Bait en 1909, rebaptisé Tel-Aviv en 1910.

${ }^{3}$ Elle se retrouve dans le titre même de l'ouvrage de N. Metzger-Szmuk (2004) Des maisons sur le sable. TelAviv : mouvement moderne et esprit Bauhaus, l'un des ouvrages de référence sur le Bauhaus à Tel-Aviv.
} 
reprend les espaces de culture : jardins, potagers, vergers ${ }^{4}$. N. Payton (1995) explique le développement de Tel-Aviv comme celui d'une ville-jardin (qui reprend tantôt le modèle anglais, tantôt le modèle allemand et notamment celui des siedlungen ${ }^{5}$ ) par des typologies architecturales modernes, comme celles du Bauhaus. En même temps, Ahuzat Bait rompt volontairement avec l'organisation de la ville arabe - Jaffa est à cette époque une gare, un port et une ville importante de Palestine ottomane - et de ses quartiers juifs ou mixtes, en s'inspirant de communautés allemandes chrétiennes implantées dans la région: Whalhalla, Whilhelma et Sarona (Schlör, 1999; Weill-Rochant, 2006). Ce nouveau quartier juif est planifié selon les règles de l'esthétique et de l'hygiène modernes « au lieu des conditions insalubres des logements de Jaffa » (Levine, 2005). Cette double nature, urbaine et architecturale, est la manifestation matérielle du projet sioniste. Or, c'est un élément difficile à appréhender par les logiques patrimoniales classiques, comme le montrent les travaux du docomomo, l'International committee for documentation and conservation of buildings, sites and neighbourhoods of modern movement.

Les dirigeants sionistes de l'époque sont convaincus que les reformes politiques ont une dimension physique et c'est pourquoi ils s'adressent à l'urbaniste et biologiste écossais Patrick Geddes en 1925 pour dessiner le plan de Tel-Aviv ${ }^{6}$. Les choix urbains qu'il opérera sont, à leurs yeux, le symbole de la culture hébraïque moderne (Helman, 2002 ; Mann, 2006) dont Tel-Aviv, qui vient d'acquérir le statut de township au sein de la municipalité de Jaffa en 1921, doit être le témoin ${ }^{7}$. Israel Zangwill (membre de l'Organisation sioniste mondiale puis fondateur de la Jewish Territorial Organisation) et les autres théoriciens d'un futur État voient en effet dans la démarche biologique de Geddes la possibilité d'émergence d'un nouvel état, sans rupture ${ }^{8}$ : une nature aménagée qui est la métaphore d'une nature humaine et urbaine où différents éléments se recomposent en s'adaptant les uns aux autres selon un métabolisme urbain structuré par la présence des espaces ouverts et végétaux (Tufano, 2016). Geddes n'hésite pas, dans ce contexte, à proposer un plan qui joue de la porosité avec la ville arabe. Il refuse les rues en cul-de-sac pour composer avec les trois communautés en présence

\footnotetext{
${ }^{4}$ Plusieurs quartiers juifs sont construits hors les murs de Jaffa dès la fin du $19{ }^{\text {ème }}$ siècle : Neve Tseddek en 1887 , puis Neve Shalom en 1890, Mahaneh Yehudah en 1903, Mahaneh Yosef en 1904 et Ohel Moshe en 1905.

${ }^{5}$ La cité-jardin anglaise trouve son fondement esthétique dans les Arts and Crafts, son inscription politique dans le socialisme utopiste de W. Morris et son enracinement socio-économique dans un modèle libéral puisque la propriété des terrains et des édifices revient à une association de particuliers ou à un mécène. La cité-jardin allemande emprunte, quant à elle, davantage le code stylistique moderne et est surtout soutenue par une vision politique proche de la gauche révolutionnaire d'un E. May ou d'un H. Meyer. Cf. P. Girard et B. Fayolle Lussac (1996).

${ }^{6}$ L'arrivée de P. Geddes s'inscrit dans une pratique courante qui consiste à transférer et à adapter en Palestine, sous mandat britannique jusqu'en 1948, un modèle urbain emprunté à d'autres contextes. Les urbanistes britanniques proposent ainsi, par exemple, de nombreux aménagements pour les bâtiments et les résidences des représentants du pouvoir, comme pour la construction et l'extension des villes existantes

${ }^{7}$ La portée politique de la ville se lit dans son nom même « Tel-Aviv » qui reprend le titre, en hébreu, du roman utopiste de Th. Herzl Altneuland («Ancien pays nouveau » en français) paru en 1902 et dans lequel il formule pour la première fois l'idée d'un foyer national juif en Palestine.

${ }^{8}$ Le travail de Geddes est nourri à la fois par sa formation en biologie et son intérêt pour l'écologie, mais également par les recherches en sciences sociales - notamment les enquêtes de terrain de F. le Play - et par l'idée de coupe territoriale empruntée à E. Reclus. C'est à partir de ces éléments qu'il proposera une matrice d'observation des villes qui relie Lieu-Travail-Famille et les inscrit dans une logique écologique.
} 
(juive, musulmane, chrétienne) et créer une ville nouvelle en continuité avec des activités ancestrales (Hysler-Rubin, 2011). C'est d'ailleurs cette capacité à mettre en relation paysage et architectures, continuités végétales et développement urbain qui fait, à l'époque, préférer Geddes à d'autres planificateurs ainsi qu'aux propositions techniques des architectes de la municipalité (principalement de culture allemande) qui maîtrisent les constructions modernes. Geddes met au point, pour Tel-Aviv, une «refondation moderne dans un jardin semimythique » (Home, 2013, p. 182) qui demande, pour être lue, de se pencher sur l'imbrication des espaces intérieurs et extérieurs, sur l'implantation des arbres et des parcelles jardinées et sur le riche appareillage végétal. C'est là que réside la force du plan de Geddes, comme dans sa capacité à résister aux épreuves du temps et, notamment, aux interventions dans les années 1930 de grands architectes modernes et de leur architecture radicalement inscrite dans le Style International (Metzger-Szmuk, 2004 ; Weill-Rochant, 2006).

Sur cette toile de fond sociale, architecturale et urbaine, deux tendances se dessinent alors quant au lien avec la terre. La première poursuit la colonisation britannique en composant avec les éléments endogènes, selon un projet politique qui essaye de concilier les cultures juive et arabe en négociant en permanence avec les formes architecturales existantes. La seconde est plus théorique. Elle s'appuie sur l'expression d'une ville-jardin aux accents réformateurs d'un projet socialiste de mixité sociale. Comment tenir compte de cette réalité, aujourd'hui, dans les critères de patrimonialisation et de protection, sachant que la reconnaissance patrimoniale se fait souvent sur des éléments matériels ? Si la question n'est pas tranchée, on peut en tout état de cause revenir sur la portée culturelle du plan de Geddes pour Tel-Aviv, pour comprendre le caractère hybride qu'une inscription de ce type demanderait et pour mieux saisir certaines incongruences dans les effets induits par cette dernière. Elle fige forcément une ville qui se voulait organique et évolutive et conduit à créer de la valeur, voire de la spéculation foncière contre laquelle $\mathrm{P}$. Geddes mettait déjà en garde dans les années 1920, et une hyperurbanisation dans le périmètre inscrit, comme à ses abords. C'est le cas du Boulevard Rothschild ; monument vivant d'une culture en pleine réforme au tournant des années 1930 (Mann, 2006) et qui a vu se développer à l'été 2011, à partir du logement à Tel-Aviv et du droit à la ville, le plus grand mouvement de protestation sociale qu'ait connu le pays depuis sa création (Rozenholc et Fijalkow, 2016).

\section{Les caractéristiques du plan à protéger}

Quelles sont, dans ce contexte, les caractéristiques du plan à protéger ? La grille urbaine de Geddes présente quatre types de rues et donne lieu à la division de base des terrains. Le choix de cette grille est assez classique, dans le sens où les plans modernes sont libres, mais ce maillage de rues qui traverse du sud au nord et croise des plus petites rues, plus espacées, donne lieu à un damier auquel se superposent des boulevards et un système de ruelles, les homeways, qui percent un système de blocs et renvoient aux polarités urbaines classiques de la cité-jardin anglaise, les crescent, square et close. Il s'agit d'une composition qui s'appuie sur les polarités vertes, soixante jardins où il décline différentes sortes d'espaces (jardins, potagers, sport, loisirs) placés au centre de chaque cœur d'îlot. Comme il l'affirme, cette 
manière d'être dans l'espace extérieur doit devenir la marque de l'habitant de ce lieu. Geddes crée ainsi une disposition spatiale très organisée, mais qui reste adaptative et évolutive.

Il y joue de linéarités-complexités avec les rues domestiques et crée des effets composites avec le passage d'espaces très urbains au centre de l'îlot par ces petites voies domestiques qu'il imaginait plantées de rosiers ou de vigne. Il choisit minutieusement les essences : arbres fruitiers, fleurs, plants de culture potagère, mûriers et oliviers qui, eux, ne résisteront pas à leur transplantation en milieu urbain. C'est dans cette relation entre les paysages extérieurs (morphologie et essences locales) et les espaces intérieurs (modernes), que l'appropriation aux lieux devra se faire et l'identité des habitants se construire. De cette manière, Geddes propose un compromis entre la garden city (ou, plus précisément un garden village au plan libre, comme il l'écrit dans son Report) et la centralité urbaine (Metzger-Szmuk, 2004). Il n'est pas question d'un agrandissement périphérique et périurbain, mais bel et bien d'une forme d'urbanisme végétal moderne dans laquelle s'incarne l'esprit des constructeurs juifs. Deux éléments formels caractérisent cette position critique. Pour ce qui est de l'architecture, le jugement de $\mathrm{P}$. Geddes est sans concession. Il en appelle à une architecture nouvelle, tout en considérant positivement les constructions qui réinventent la tradition locale. Sur ce point, les réalisations des années suivantes constitueront une vraie rupture conceptuelle. Au regard de ses choix stylistiques, le modèle de Geddes est en effet plus proche de la modernité des Arts and Crafts que de celle du Bauhaus, même si c'est bien dans la conjugaison avec cette dernière que réside le caractère unique et patrimonial de Tel-Aviv.

Ainsi, quand Arieh Sharon, issu de l'école du Bauhaus, fera le choix de la Palestine, il s'attachera à donner forme à ce plan en s'inscrivant dans la modernité généalogique de TelAviv. Il le fera cependant en rompant parfois, de manière subtile, avec le modèle de Geddes qu'il considère comme une itération méditerranéenne d'un produit confectionné pour et par le monde anglo-saxon, comme il le dit avec ironie : "Thus it would be a typical, style dormitory garden city, although located on the Mediterranean " (Sharon, 1976 : p. 47). L'engagement d'A. Sharon le porte à promouvoir une relation au sol plus politisée et plus libre: un collectivisme politique israélien de la propriété étatique des sols, des logements collectifs et de l'insistance sur les espaces publics qui oppose à l'expression « la forme suit la fonction », «la forme suit l'histoire ». L'expression de cette histoire d'une transformation urbaine fortement marquée par la modernité du Bauhaus et teintée d'un idéalisme coopératif est une architecture ouverte sur l'espace vert grâce aux pilotis qui soutiennent la plupart des immeubles des quartiers résidentiels de Tel-Aviv et sont devenus l'image de marque de la ville (Sharon, 1976, p. 58). Les jardins de Geddes deviennent des espaces verts et, à l'idéal de la nature industrieuse qui permet de réinventer une tradition d'échange et de commerce dans l'espace public, succède une vision de l'espace ouvert sain. Sharon (1976, p. 58) soulignera la nécessité d'avoir «plus d'arbres pour la ville hébraïque » pour raconter une ville propre. S'il est indéniable que le plan Geddes impose des gabarits, les architectes modernes, tout en faisant preuve d'imagination pour s'y intégrer, vont donc marquer d'un sceau moderne l'image de Tel-Aviv. Ils créent, à partir du «blanc» et des «pilotis », un langage moderne qui s'adapte au climat et donne lieu à une forme singulière. Ainsi, pendant un certain temps, 
paradoxalement, l'élément de pérennité urbaine sera davantage le plan (grâce à sa capacité d'adaptation) que l'architecture ayant subi des modifications et des altérations profondes.

Dans le cadre de la construction de la question patrimoniale à Tel-Aviv, il aurait ainsi été possible de proposer un double registre de protection et de classement. Le premier portant sur le housing block permettrait d'élargir le modèle à de plus grandes parties de la ville, et, le second, en complément, portant sur des interventions conservatives de l'architecture. Patrimonialiser dans ce contexte demande en effet un traitement au cas par cas (WeillRochant, 2006), avec le recours à des critères plus qualitatifs que quantitatifs et une sollicitation permanente des données historiques pour rétablir la généalogie du garden village de Geddes et des typologies architecturales du Bauhaus.

\section{Patrimonialiser les modernités : une reconnaissance patrimoniale en urgence}

Faire le choix de patrimonialiser est toujours une affirmation de politique culturelle et une orientation en matière de choix des priorités dans la vie urbaine. Derrière un bâtiment à protéger, se tisse toujours un discours qui vise à mettre en lumière un élément jusqu'à là passé inaperçu - ou considéré comme anti-esthétique et soumis à des risques de modifications et de destructions - et qu'un groupe restreint impose comme patrimoine. Au fur et à mesure que la notion s'est étendue à des objets urbains, la patrimonialisation a dû composer avec des ensembles auxquels il était impossible d'appliquer des règles de protection destinées à un objet car, comme l'avait déjà écrit A. Riegl (1903), la valeur d'un immeuble à protéger ne peut être pensée de manière disjointe de son usage. De surcroît, la manière même de faire reconnaitre en tant que patrimoine un ensemble urbain ne peut plus se faire séparément de la vie que s'y déroule (Melot, 2012). Parfois, c'est dans le conflit entre discours officiel et action d'appropriation de cet élément qu'on voit surgir la notion patrimoniale (Le Goff, 1997 ; Leniaud, 2001 ; Walter, 2004 ; Béghain, 2012). A ces questions, s'ajoute celle de la notion même de patrimoine évoquée dans cette protection, car, in fine, le choix des objets se fait sous une pression politique dont les finalités sont essentiellement économiques (Melot, 2012). De surcroit, la reconnaissance patrimoniale accordée aux architectures modernes est une pratique récente (les premiers bâtiments classés datent des années 1980) dont les critères sont encore en train de s'écrire. Les questions qui se sont posées lors des premières inscriptions portaient sur le langage de cette architecture - qui affirme une rupture radicale vis-à-vis d'éléments historiques - et sur la manière de le protéger ou d'y intervenir. Or, l'expérience de 30 ans d'activité patrimoniale a montré qu'outre les problèmes de restauration technologique (qui sont au centre de débats déontologiques quant à la préservation des éléments d'origine), l'aspect vivant de l'ensemble est souvent sacrifié au profit d'une protection esthétique fondée sur la reconnaissance de la cohérence architecturale (Casciato et D’Orgeix, 2012).

Tel-Aviv raconte tout cela. Non seulement, pendant longtemps on n'a accordé, en Israël, de valeur qu'aux seules antiquités, mais le classement de l'Unesco de Tel-Aviv a déclenché de nouvelles problématiques. La connivence avec ce patrimoine y était en effet d'autant plus hybride que la méditerranéité de cette ville (Rozenholc, 2014) avait, en quelque sorte, repris 
le dessus en juxtaposant les modes de vie et les styles composites. Le discours moderne avait donné lieu à Tel-Aviv à un langage de synthèse, en proposant une forme nouvelle qui ne permet pas d'identification univoque : le langage architectural et urbain portait la volonté d'effacer les aspérités conflictuelles que produisent différentes identités et appartenances, communautaires et, en l'occurrence, largement issues des vagues d'immigration successives qui faisaient déjà la ville. Dans ce sens, la Ville blanche de Tel-Aviv est, de par son plan même, un compromis qui devait raconter la nécessaire alliance politique liée à la naissance d'une ville nouvelle : la première ville hébraïque des temps modernes, établie au sein d'une municipalité arabe musulmane et chrétienne (Jaffa) en contexte colonial (britannique). Or, lorsque les mémoires convergent, les lieux cristallisent des conflits et la mise en récit de la mémoire devient un support « objectivant» de patrimoine. La reconnaissance, puis la mise en patrimoine, construit le regard et, par-là, transforme l'objet patrimonialisé. Pour Tel-Aviv, la question de la capacité de la mise en patrimoine de l'Unesco à intégrer les différences se pose même si, en réalité, un double registre de patrimonialisation (Davallon, 2015) existe : d'une part, celui matériel qui se construit par la reconnaissance de la valeur architecturale ; d'autre part, celui qui tient aux éléments immatériels (caractère hybride de l'urbanisme végétal, pratiques de l'espace ouvert, cohabitations et mitoyennetés dans le quartier et à l'interface avec d'autres quartiers). Les politiques et les modalités de de patrimonialisation et de conservation peuvent-elles être aussi évolutives que ces objets hybrides ? Marquent-elles au contraire davantage la rupture entre la cristallisation d'un morceau de ville et le reste de l'urbain ? À Tel-Aviv, le choix de mettre en exergue le seul patrimoine matériel par son architecture, qui plus est uniquement représenté par le Bauhaus, fige non seulement l'objet patrimonialisé mais aussi l'image qui en est transmise. Cette dernière devient un « label de qualité » qui peut fonctionner comme levier de développement territorial mais attise aussi, comme le dit M. Rautenberg (2015), les convoitises économiques.

Les raisons de l'inscription sont pourtant légitimes. La dégradation de la ville moderne imposait un entretien qui ne semblait pouvoir se faire qu'à travers une patrimonialisation appelée de toute urgence par des personnalités de renom. Dès 1959, à l'occasion des célébrations des 50 ans de la ville, une tentative de mise en valeur est proposée, mais il faudra attendre l'exposition que Michael Levin met sur pied au Musée d'art de Tel-Aviv, en 1984, pour commencer à considérer sérieusement la nécessité de conservation de la Ville Blanche. Intitulée White City. International Style Architecture in Israel, cette exposition marque le début d'un intérêt, aussi bien local qu'international, pour la ville. La subtilité de l'exposition, en deux volets (l'un avec les photographies de Judith Turner et l'autre avec les descriptions de Michael Levin) réside dans la capacité à avoir artialisé (Roger, 1997) le regard porté sur ce quartier et nommé cet objet : on invente ici un sigle (Ville blanche) et une vision géométrique de formes abstraites photographiées qui caractérise la modernité dont il est question ${ }^{9}$. Ce passage par la photographie est fondamental, car il permet d'extraire cette partie de la ville de sa quotidienneté, de sa banalité, et d'en donner une image stylisée ; des volumes élégants, qui donnent à voir sur leurs surfaces une image réussie de la construction culturelle de la ville

\footnotetext{
${ }^{9}$ Sur cette question, voir aussi Y. Fisher (1984) et M. Azaryahu (2007).
} 
(Mann, 2006). Il induit cependant deux autres effets : d'une part, la déconstruction du lien au territoire, la « déterritorialisation » de l'architecture de Tel-Aviv, qui permet de construire une notion abstraite de valeur; d'autre part, la mutation de l'antériorité du récit patrimonial en un discours sur le produit monumental, avec l'émergence d'une «patrimondialisation» qui induit un développement et une consommation touristique inattendue (Gravari-Barbas, 2012).

Cette exposition pionnière déclenche ensuite un intérêt qui suscitera des études académiques de plus en plus nombreuses sur les «auteurs» de ces architectures-œuvres d'art. En 1994, Tel-Aviv accueille la conférence de l'Unesco intitulée Pilgrimage to the White City qui institutionnalise officieusement ce nom et surtout réveille l'attention internationale sur ce type de patrimoine ${ }^{10}$. Comme le rappelle R. Ingersoll (1995), c'est à cette date que l'on prend véritablement conscience de l'existence d'un ensemble de bâtiments dit du Bauhaus qui, pour la première fois, n'étaient pas isolés comme à Dessau, mais constituaient une ville à part entière, un noyau qui se distinguait par l'unité de conception et réalisation des architectures (entre 1930 et 1940) et la capacité plastique de son maillage à suivre le contexte environnant d'une ville mouvante. Levin est ainsi le pionnier de la reconnaissance culturelle, le déclencheur d'un mouvement de sensibilisation conduit également par A. Shamay, l'un des ingénieurs de la municipalité de Tel-Aviv. Cette reconnaissance du patrimoine est particulièrement importante pour Israël puisqu'elle marque aussi celle de la nation. Israël, tenu à l'écart des décisions et réunions internationales, trouve là, par les organisations mondiales pour la culture, une légitimation. Localement, il est important de souligner que cette reconnaissance est également portée par d'autres acteurs et, en premier lieu, par l'artiste Dani Karavan mu à la fois par sa connaissance architecturale et historique de la ville, des questions patrimoniales européennes (il travaille alors à Florence) et par son entregent politique. Il emmène, dans son sillage, des universitaires pour préparer le dossier de classement pour l'Unesco avec, à leur tête, Nitza Metzger-Szmuk avec qui il collabore déjà ${ }^{11}$.

En même temps que se constitue le dossier d'inscription, et devant l'urgence de l'intervention, se pose pour la première fois la question des choix de restauration, de réhabilitation ou de conservation et du périmètre à inscrire. Ces questions étaient tout à fait nouvelles au moment du montage du dossier car, la patrimonialisation du moderne était inédite. D’ailleurs, alors que d'habitude on classe un patrimoine dont on a la connaissance historique, c'est l'inverse qui s'est passé à Tel-Aviv, car la mise en patrimoine a marqué le démarrage de l'étude systématique des architectes qui ont construit Tel-Aviv. Les recherches de B. Hyman (1994) et R. Home (2013) complètent les études en cours et N. Metzger-Szmuk entreprend une réflexion sur les types de conservation possibles, tout en ouvrant la discussion avec les acteurs de l'urbanisme et les propriétaires qui craignent alors que l'inscription ne bloque le développement de la ville et ne leur soit trop coûteux. S'amorce alors un débat qui porte, d'une part, sur la stratégie de conservation et, d'autre part, sur l'échelle territoriale du site classé $^{12}$ : les implications et les imbrications de ces deux éléments seront à l'origine de

\footnotetext{
${ }^{10}$ La conférence se tint du 22 au 27 mai 1994.

${ }_{11}^{11}$ Entretien téléphonique de juillet 2014 avec D. Karavan.

${ }^{12}$ Entretien téléphonique de juillet 2014 avec N. Metzger-Szmuk.
} 
polémiques dont les effets restent visibles aujourd'hui. Entre identité, authenticité et intégrité, se nouent des questions déontologiques, politiques et économiques dont les différents acteurs mesurent différemment l'importance, selon qu'il s'agisse d'une institution supranationale (Unesco, Icomos), d'une volonté politique impactant la visibilité d'une ville et les enjeux touristiques (la municipalité avec à sa tête Ron Huldaï élu en 1998 pour la première et pour la cinquième fois consécutive le 30 septembre 2018), d'urgence de gestion locale et les innombrables intérêts particuliers qu'un site classé peut engendrer (Frey, 2011).

\section{L'inscription par l'Unesco comme levier de conservation et de stratégie urbaine}

Le dossier est soumis dans la catégorie culturelle du classement Unesco. En s'appuyant sur l'Article 1 de la Convention du Patrimoine Mondial (1972) ${ }^{13}$, est demandée la reconnaissance en tant qu'ensemble de bâtiments, en précisant qu'il s'agit d'une aire urbaine représentative d'une nouvelle ville du $20^{\text {ème }}$ siècle (OG, 1999, p. 27 : iii). En mars 2003, l'Unesco se prononce favorablement et inscrit la ville au titre des critères II et IV de la Convention de $1972^{14}$. Pour le critère II, ils reconnaissent que la « Ville blanche de Tel-Aviv est une synthèse d'une valeur exceptionnelle des diverses tendances du mouvement moderne dans l'architecture et de l'urbanisme dans la première partie du $20^{\text {ème }}$ siècle. Ces influences ont été adaptées aux conditions culturelles et climatiques du lieu, ainsi qu'intégrées aux traditions locales ». Pour le critère IV : « La nouvelle ville de Tel-Aviv est un exemple remarquable de l'architecture et l'urbanisme nouveaux du $20^{\text {ème }}$ siècle, adaptés aux exigences des contextes culturel et géographique particulier ${ }^{15}$.

Malgré cette réussite, l'acceptation est assortie de recommandations des experts du Conseil international des monuments et des sites (ICOMOS) qui insistent sur la nécessité de protection, sachant que l'Unesco n'en exerce pas en tant que telle. En attendant qu'une législation nationale soit créée, le suivi du développement urbain et la supervision, voire le contrôle, des changements dans les bâtiments existants sont recommandés ${ }^{16}$ : même en l'absence d'une législation particulière sur le patrimoine récent, la réglementation d'urbanisme doit protéger le patrimoine, tout en sachant que c'est avec le classement de l'Unesco qu'un vaste chantier international s'est ouvert pour mieux comprendre sur quoi porte l'exceptionnalité du patrimoine moderne et comment y intervenir (Jokilehto, 2006). Le comité, au moment du classement, reconnait l'existence de surélévations dans la zone protégée et dans la zone dite tampon, mais préconise l'interdiction de toute nouvelle surélévation ou édification de bâtiments. Enfin, pour les experts il est indispensable de vérifier

\footnotetext{
${ }^{13}$ Extrait de l'article 1 de la Convention Unesco de 1972 : «Aux fins de la présente Convention sont considérés comme 'patrimoine culturel' : les ensembles, groupes de constructions isolées ou réunies, qui, en raison de leur architecture, de leur unité ou de leur intégration dans le paysage, ont une valeur universelle exceptionnelle du point de vue de l'histoire, de l'art ou de la science ».

${ }_{15}^{14}$ Le critère I n'a pas été présenté par l'État partie.

15 Selon l'évaluation et les recommandations de l'Unesco publiées en ligne en mars 2003, p. 56-74, http://whc.unesco.org/archive/advisory body evaluation/1096.pdf.

${ }^{16}$ Le document de l'Unesco rappelle également qu'il faut améliorer, là où s'est possible, le contrôle des changements du tissu existant (2003, p 61).
} 
la cohérence entre le plan d'aménagement et le plan de conservation, afin de garantir la protection de l'ensemble urbain.

Dès la prise de conscience de la valeur culturelle de ces immeubles, des interventions avaient été faites pour les conserver et protéger des altérations qu'ils avaient subies (surélévations et interventions sur les façades). Dans les années 1990, le Conservation Team de la municipalité de Tel-Aviv, sous l'égide de N. Metzger-Smuk, mène une étude des bâtiments (documentation systématique, histoire iconographique, suivi de leur évolution) et une réflexion sur les modalités de conservation. Dans cette période, 1149 bâtiments sont listés et font l'objet d'une intervention dans la logique des techniques constructives, des matériaux et des détails artisanaux d'origine. Un document intitulé Guiding Principles for the Care and Conservation of listed buildings (Conservation Plan, TA 2650 B) permet d'établir le niveau de restauration et détaille ces éléments. Le principe de restauration adopté sera d'un pragmatisme qui met parfois à mal la conservation patrimoniale. Lorsque les ajouts sont de mauvaise qualité, il faut retrouver l'état d'origine des bâtiments; lorsque les ajouts ne déqualifient pas la valeur architecturale du bâtiment, il est possible de les garder ou d'effectuer des interventions de compromis entre le retour à l'état d'origine et la préservation des strates successives. Dans tous les cas, nombre de bâtiments inclus dans la liste à préserver se verront octroyer le droit de construire des étages supplémentaires (souvent en retrait des façades) de manière à payer le coût de rénovation des bâtiments. En réalité, la ville n'a pas les moyens financiers de son programme de réhabilitation (Cohen, 1999).

Document 2: La préservation des bâtiments comme prétexte de façade ? 


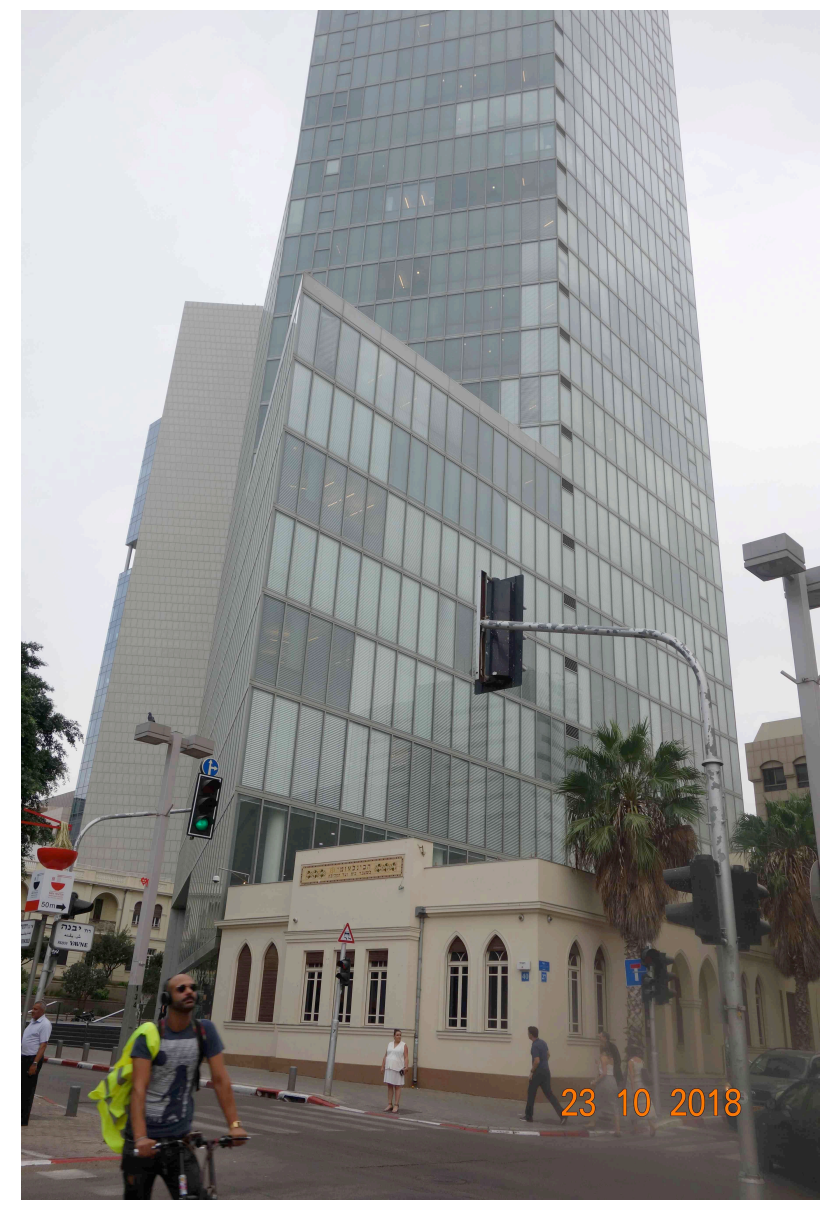

Légende : Commencée en 2003, au moment de l'inscription de Tel-Aviv au patrimoine mondial de l'Humanité, cette tour de bureaux de 132 mètres de haut est l'œuvre des New-Yorkais Pei, Cobb, Fried \& Partners (les concepteurs de la pyramide du Louvre) et de Nir-Kotz Architects. La «First International Bank Building» se situe au coin du boulevard Rothschild et de la rue Yavne et est achevée six ans plus tard en 2009. Elle est construite de sorte à laisser la vue sur plusieurs bâtiments qui devaient être préservés et dont les façades ont été rénovées pour l'occasion mais qui semblent, dès lors, tout à fait hors contexte: la Levin House, la Rivka Grinwald House et la Community Committee's House.

Crédits : C. Rozenholc, octobre 2018

Au début des années 2000, la municipalité de Tel-Aviv créée néanmoins un Département de Conservation d'une dizaine de personnes qui travaillent de concert avec le Département d'urbanisme. C'est également au sein de la municipalité que sont formulées, en 2001, les nouvelles directives de conservation et restauration dans un document intitulé, en anglais, Instructions for Care and Conservation of Listed Buildings. Le Département de Conservation s'occupe de la préservation d'Ahuzat Bait, le noyau historique de Tel-Aviv (dont par ailleurs tous les bâtiments d'origine ont été détruits), la réhabilitation de Jaffa, des bâtiments de la 
Ville blanche et, plus récemment, des architectures construites après 1948. Comme le dit Jérémy Hoffmann, le directeur du Département dans un entretien de $2011^{17}$ :

«Nous voulons grâce à notre travail préserver l'histoire de l'État, l'histoire de la ville, souligner l'apport de l'architecture au social. Il faut aussi comprendre la valeur architecturale de quartiers (...) qui sont créés hors des limites définies par le fameux plan Geddes. D'autres découvertes restent à faire, il faut élargir la notion de patrimoine (...) On assiste à une ouverture des termes patrimoine et conservation qui englobent désormais d'autres champs d'activité. Cela peut comprendre aussi certaines plantes, des régions vertes, le patrimoine culturel et aussi l'architecture palestinienne, comme les biara, les batei béer, les maisons-puits notamment à Jaffa ».

Outre le nécessaire élargissement de la notion de patrimoine, on comprend la nécessité de composer avec le dynamisme de la ville. Ainsi, il est intéressant de voir comment la justification d'une certaine tolérance dans l'application des règles patrimoniales, tout particulièrement la présence de tours dans le champ de visibilité du site protégé, ne semble pas contradictoire aux yeux des décideurs institutionnels. Dans cet entre-deux (entre la règle patrimoniale et la logique de l'évolution) se situe le cas particulier de la zone-tampon qui n'est pas réglementée. Sa gestion dépend de la municipalité qui a tout intérêt (économique) à la développer, déterminant ainsi une forme d'urbanisme de disruption.

«La ville bouge, la ville change. De grands projets sont en cours (...) Ce travail de conservation n'est pas figé, il doit se faire aussi dans un esprit de renouvellement. Nous voulons que cette place ne soit pas réservée à la culture d'une élite mais à tous ». Aux questions concernant un certain état d'abandon des bâtiments du site classé, le directeur du Département répond: "l'urgence de l'entretien ne s'est pas encore développée chez nous, c'est un problème culturel national ».

Il convient de s'arrêter sur ce point précis de la surélévation des bâtiments de la zone classée qui ne font pas l'objet de la protection maximale (dans un périmètre Unesco pourtant relativement petit) et la construction de bâtiments hauts dans la zone-tampon qui entoure le périmètre. Cela s'avère nécessaire, car Tel-Aviv est une ville dont le tissu se renouvelle rapidement, et ce depuis sa création. C'est également, à l'instar d'autres villes méditerranéennes, un espace urbain où les surélévations sont courantes et pour lesquelles, au moment-même de l'inscription, les propriétaires recevaient encore des permis de construire. Cette permissivité permettait en effet une évolution sans changement radicale des bâtiments, tandis que le contexte actuel marque une cristallisation du périmètre de haute protection patrimoniale et une pression qui se concentre sur les zones tampon. Elle crée, à l'échelle urbaine, une rupture morphologique et sociologique. La question peut donc aussi être posée en termes de politiques patrimoniales et de la nécessité de les faire évoluer. La volonté du

\footnotetext{
17 «Tel-Aviv, esprit d'ouverture - Interview avec Rachel Samoul», Continuum - la revue des écrivains israéliens de langue française, 2009, $\mathrm{n}^{\circ} 6, \quad \mathrm{http}: / /$ kefisrael.com/2011/02/25/tel-aviv-esprit$\mathrm{d} \% \mathrm{E} 2 \% 80 \% 99$ ouverture/.
} 
plan Geddes était une continuité d'expansion de la ville, une densification, mais le tout dans un gabarit qui ne concédait pas la possibilité de faire des constructions hors de l'échelle urbaine. Il est donc légitime de questionner la politique qui préside à ces choix, sachant que l'inscription semble avoir, paradoxalement, accentué le développement vertical de la ville. En témoigne la photographie ci-dessous prise au coin de la rue Herzl et du boulevard Rothschild.

Document 3 : Labellisation Unesco et accélération du développement vertical de la ville

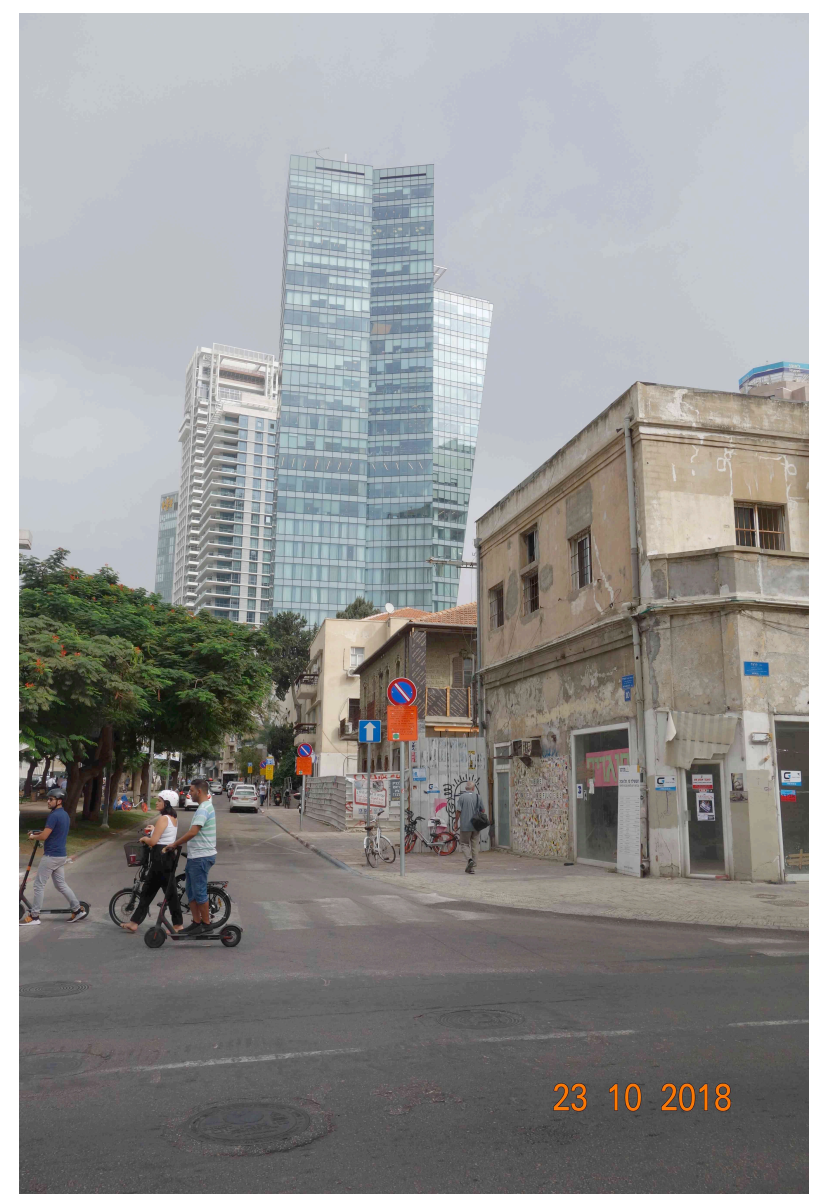

Légende : cette photographie met en évidence un contraste d'échelle de plus en plus marqué au sein de la ville avec des hauteurs spectaculaires, et sans commune mesure, avec celles des interventions et des surélévations des bâtiments réalisées jusqu'au tournant des années 2000. Bien qu'anarchiques, ces dernières - et leur gabarit très modeste - étaient le reflet de l'évolution d'un quartier et de la composition socio-économique de sa population. Or, le surplus de valeur produit par le label Unesco depuis 2003 sert de levier à la politique menée par la municipalité pour hisser la ville au premier rang des «Global Cities ». S'y installe alors un type de développement lui aussi «mondialisé » et dont les intérêts outrepassent ceux du quartier, comme lieu de vie. La rupture urbaine ainsi opérée est le reflet d'une rupture d'avec la cohabitation de plusieurs cultures qui composait un tressage raisonnable entre la règle urbaine et le développement quelque peu désordonné de la vie qu'elle permettait.

Crédits : C. Rozenholc, octobre 2018. 


\section{Mondialisation, patrimonialisation et homogénéisations}

L'inscription au Patrimoine mondial de la Ville blanche a eu un effet homogénéisant sur l'élément culturel et architectural qui avait permis de la distinguer et, dans le même temps, sur les usages et pratiques urbaines. Pour le dire de manière un peu abrupte, le patrimoine s'est ainsi trouvé vidé de son contenu politique et social, au profit d'une valeur esthétique et commercial. D'ailleurs, d'autres réhabilitations en cours à Tel-Aviv montrent les tensions entre une élite culturelle qui procède à la reconnaissance de la valeur, les réhabilitations en décalage avec les usages précédents d'un bâtiment et l'échec d'un vrai débat public qui pourrait permettre la construction d'une protection patrimoniale pensée à plusieurs voix (Mualam et Sybblis, 2016). La transformation de la Tahana, la gare ottomane de Jaffa, les réhabilitations des ports de Tel-Aviv et de Jaffa, voire le déplacement des maisons en bois du quartier de Sarona en sont autant d'exemples. Ces dernières, construites par une communauté allemande issue du mouvement luthérien de la Société du Temple (la Tempelgeselschaft) à la fin du XIXe siècle, ont en effet été démontées et réassemblées pour constituer, dans l'un des quartiers nord de Tel-Aviv, une autre des nouvelles centralités commerciales de la ville.

Évacué à la fin des années 1990 en vue de sa privatisation, le port de Jaffa - l'un des espaces les plus symboliques de la ville arabe (Cohen, 2000) et de l'arrivée en Palestine des grandes vagues d'immigration juives - est concédé en 2007 par l'administration israélienne (la Israel Land Administration), propriétaire du terrain, à la municipalité de Tel-Aviv. L'idée est alors de faire du port de Jaffa, à la manière du port de Tel-Aviv au nord de la ville (Ha'namal TelAviv en hébreu) une dizaine d'années plus tôt, un vaste espace culturel et commercial. Le port de Jaffa doit lui aussi pouvoir redevenir une valeur immobilière et un espace d'investissements. Il doit pouvoir «retrouver sa magie d'antan» comme le titrait en novembre 2007 le journal Haaretz $^{18}$, non sans interroger sur la portée de travaux qui oscillent entre réhabilitation physique, transformation des pratiques et un inévitable effacement des mémoires. Non loin de là, et en même temps que celle du port, est entreprise la rénovation de la station de chemin de fer ottoman située au sud de la ville. Cette gare de train de la ligne Jaffa-Jérusalem avait été inaugurée en 1892 puis fermée en 1948 avant de disparaître du paysage urbain. Après la conquête de Jaffa, le site déclaré « zone militaire » avait en effet été bouclé par des grillages, des bâches et bientôt recouvert par la végétation. Rouverte au public en 2009, après plusieurs années de travaux qui en ont fait un «espace culturel, de divertissement et de loisirs » (voir le document 4 ci-dessous), sa réhabilitation a donc rendu à la ville un site « retiré » du paysage depuis soixante ans. Mais quelle peut être la réception de ce bâtiment profondément transformé par ses nouveaux usagers ? Permet-il à ceux qui viennent s'y divertir de saisir le sens de son implantation dans un espace aujourd'hui évidé ; le quartier de Manshia qui, avant sa destruction lors de la conquête de Jaffa par les forces armées juives en 1948, occupait le bord de mer? La présence de cette gare, ses murs, un wagon et quelques rails, permet-elle d'en comprendre la valeur patrimoniale, symbolique et historique : celle du rayonnement de Jaffa à la fin du XIXe siècle, son insertion dynamique

\footnotetext{
${ }^{18}$ « Bringing the magic back to Jaffa », Haaretz, édition anglaise en ligne du 22 novembre 2007.
} 
Document 4 : La gare ottomane de Jaffa en travaux, avant sa réouverture au public

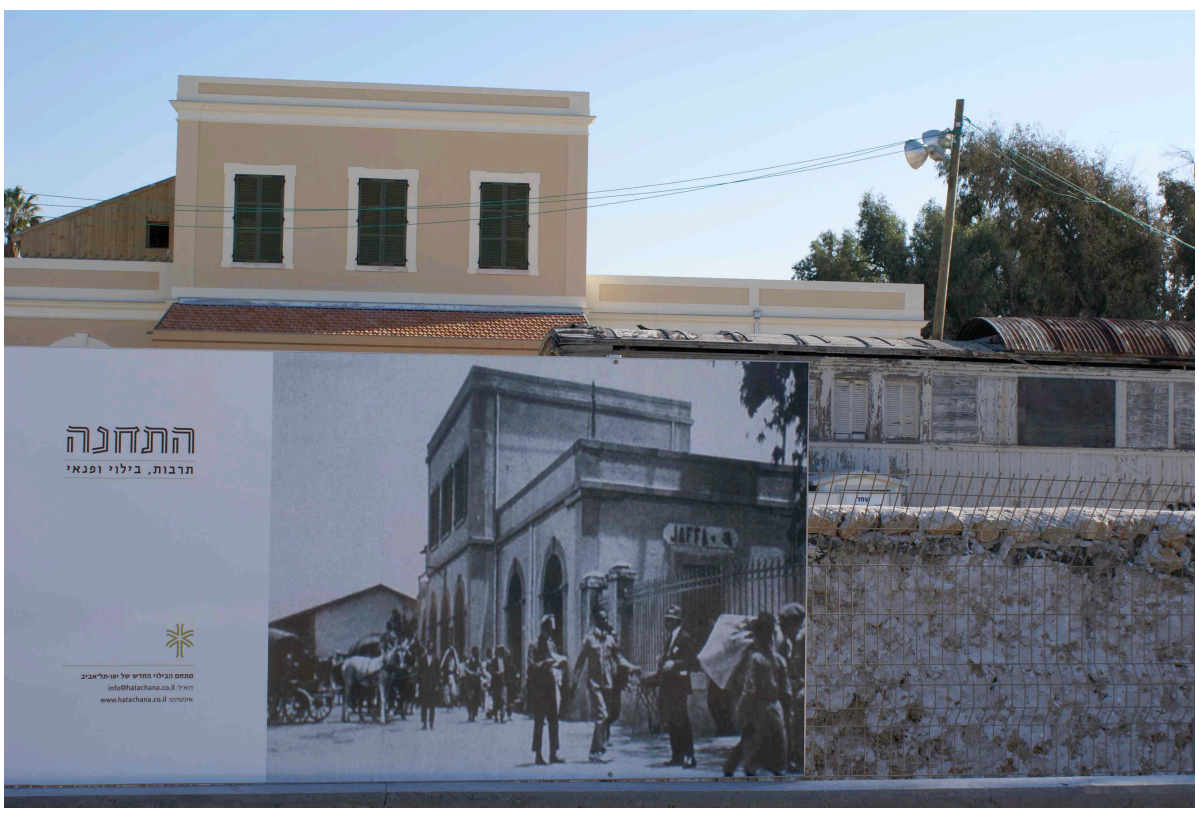

Légende: Avant sa réouverture au public en 2009, un panneau, au premier plan de l'image, communique sur les transformations en cours de l'ancienne gare de Jaffa de manière particulièrement sommaire : quelques mots qui accompagnent une photographie d'archive. Celle-ci représente la gare de Jaffa alors en activité et donne à voir, outre le bâtiment principal (dont on aperçoit la façade déjà rénovée à l'arrière plan de l'image), des voitures à cheval et des usagers (voyageurs, commerçants ?). A gauche de cette photographie, trois niveaux de texte complètent «l'explication »: "ha'tahana », «La gare» en hébreu, inscrit en gros caractères, puis «tarbout, bilouï, penaï», «culture, divertissement, loisirs » en plus petit. On notera l'insistance sur l'unicité de la gare ( $L a$ gare) et sur le caractère culturel (le premier des trois termes) de sa transformation. Plus bas encore, sous le sigle de l'opérateur des travaux, une phrase qui décrit le projet à venir : " mitham bilouï hahadash shel Iafo-Tel Aviv », " le nouvel espace de divertissements de Jaffa-Tel-Aviv». Il est intéressant de noter que cette phrase met l'accent, de manière inhabituelle, sur Jaffa plutôt que sur Tel-Aviv, dans une municipalité dont l'intitulé complet est Tel-Aviv - Jaffa. Derrière le panneau, apparaît le grillage qui entoure alors le site, devant un mur d'époque puis, au second plan, un wagon en l'état avec ses volets en bois et son toit de tôle. À l'arrière plan, le bâtiment principal de la gare qui semble avoir été restauré à l'identique.

Crédits : C. Rozenholc, janvier 2008.

Le classement met donc sous cloche, directement ou indirectement, ponctuellement des bâtiments manifestes, ou leur façade, et produit, tout en la transformant, une forme de modernité mondialisée qui profite du caractère vitaliste de Tel-Aviv et du métissage dont elle a de tout temps été le lieu (Tzfadia et Yacobi, 2011) pour produire des ruptures violentes du tissu urbain. Mettre sous cloche le cœur de la Ville blanche est aussi un acte de patrimonialisation conservative cristallisante qui pourrait induire le renforcement de la rupture entre deux Palestine construite par le mouvement sioniste au début du XXe siècle (Le Vine, 
$2005)^{19}$, et ce avec des effets paradoxaux en chaîne : la demande touristique centrée sur ce site et, en général, sur la Ville blanche, européenne et mondiale, internationale, et l'oubli volontaire ou non d'autres quartiers du sud de Tel-Aviv, ces anciens quartiers juifs de Jaffa, à la tradition plus hybride ; la pression immobilière sur les zones tampon et le désintérêt pour une cohérence harmonieuse avec les quartiers mixtes qui jouxtent ces sites; la protection maximale du bâti moderne du périmètre Unesco et la légitimation de nouvelles tours qui ceinturent le périmètre et le mettent à distance du reste de la ville. Aujourd'hui, l'inscription au patrimoine mondial de l'Humanité de la Ville Blanche moderne, dans un périmètre défini, valide indirectement, voire renforce cette idée de la séparation et ses effets socio-urbains. Se créée aussi une plus-value touristique qui renforce les écarts au lieu de les réduire et transforme ce quartier multiforme en un site de tourisme et de commerce qui enterre la complexité urbaine (Alsayyad, 2001 ; Rofe, 2009).

\section{Conclusion}

N'y a-t-il pas là contradiction dans les termes entre la proposition de Geddes pour Tel-Aviv, comme "ville vivante », évolutive, inscrite dans un contexte culturel mixte et des conditions climatiques singulières et la patrimonialisation de la seule Ville blanche ? Contradiction d'autant plus criante que le reste de la ville entretient ce caractère contradictoire qui en fait la spécificité (Almog, 2006 ; Allwel et Kallus, 2008). Les effets sur le tissu social sont tout aussi forts avec la promotion d'une ville dotée d'une architecture qui s'affirme de plus en plus comme libérale, au détriment de la population qui n'y trouve plus à se loger. Par ailleurs, la convergence grandissante entre Tel-Aviv et la Ville blanche (Tel-Aviv devient la Ville blanche), peut aussi être décrite comme le fait d'une certaine confusion entre mémoire(s) et histoire, dans les discours politiques, médiatiques et populaires. Son corollaire le plus évident et la mise au ban d'autres mémoires et d'autres quartiers de la ville, avec des contrastes particulièrement marqués entre la Tel-Aviv Ville blanche et les quartiers sud de la ville, ces anciens quartiers juifs de Jaffa et la ville de Jaffa même formant un ensemble (lui même non homogène) auquel l'architecte israélien S. Rotbard (2003, 2015) se réfère comme étant le pendant de la Ville blanche : une Ville noire associée aux populations palestinienne, arabe israélienne et juive dite «orientale» qui profite peu des retombées économiques de la patrimonialisation et de la mise en tourisme de Tel-Aviv, alors même qu'ils font partie intégrante de l'histoire du développement de la ville au début du siècle. Des quartiers que le plan Geddes imaginait ensemble, se retrouvent tel Florentine ou Neve Sha'anan à la marge.

Peut-on dire pour autant que la patrimonialisation de la Ville blanche et l'identification de Tel-Aviv à cette mémoire là servent la promotion de la ville comme ville dont l'exceptionnalité tient à son origine européenne ? Elle est certes l'héritage le plus important de la mise en œuvre des idées du Bauhaus, conjuguée à l'urbanisme des cités-jardins. Mais aborder Tel-Aviv de cette manière, en soulignant sa dimension internationale, mondiale,

\footnotetext{
${ }^{19}$ Mark Le Vine (2005) montre, à travers l'analyse de différents documents, comment cette séparation traduisait l'idée qu'il existait deux Palestine, l'une juive, moderne et progressiste, l'autre arabe, traditionnelle et arriérée.
} 
européenne et méditerranéenne plutôt qu'israélienne et proprement moyen-orientale (Rozenholc, 2014), n'est-ce pas aussi renforcer ou jouer du sentiment que Tel-Aviv n'appartient pas tout à fait à son contexte : un espace israélo-palestinien déchiré par un conflit non-résolu. Promouvoir Tel-Aviv comme Ville Blanche, dans le périmètre restreint validé par l'Unesco, c'est aussi participer à sa déterritorialisation, et ce à l'encontre du plan inclusif de Geddes.

\section{Bibliographie}

Alfasi N. et Fenster T. (2005). «A tale of two cities : Jerusalem and Tel Aviv in an age of globalization », Cities, vol. 22, n5, p. 351-363.

Allwel Y. et Kallus R. (2008). «Public-space heterotopias : heterotopias of masculinity along the Tel Aviv shoreline », M. Dehaene et L. De CAuter, Heterotopia and the city public space in a postcivil society, Routledge, Londres et New-York, p. 191-201.

Almog O. (2006). The Sabra: the Creation of the New Jew, Berkeley, University of California Press, 328 p.

Alsayyad N. (2001). Global Norms and Urban Forms in the Age of Tourism, London, Routledge, $308 \mathrm{p}$.

Azaryahu M., (2007). Tel Aviv. Mythography of a city, New York, Syracuse University Press, $319 \mathrm{p}$.

Batty M. et Marshall S. (2009). «The evolution of cities: Geddes, Abercrombie and the new physicalism », TPR, Centenary paper, vol. 80, nº, p. 551-574.

BÉGHAin P. (2012). Patrimoine, politique et société. Paris, Presses de Science Po, 162 p.

Casciato M. et D'Orgeix E., (2012). Architectures modernes. L'émergence d'un patrimoine, Wavre, Mardaga, 195 p.

Charney I. (2007). « The Politics of Design : Architecture, Tall Buildings and the Skyline of Central London », Area, vol. 39, n², p. 195-205.

Cohen N. (1999). Urban Conservation, Cambridge, The MIT Press, 359 p.

CoHen R. (2000). « Jaffa, port de Jérusalem au XIXe siècle », Les Cahiers du judaïsme, nº7, pp. 44-55.

Cometti J.-P. (2015). Conserver/Restaurer. L'œuvre d'art à l'époque de sa préservation technique. Paris, Gallimard, coll. NRF, 300 p. 
Davallon J. (2015). « À propos des régimes de patrimonialisation : enjeux et questions » Patrimonialização e sustentabilidade do património: reflexão e prospectiva, nov 2014, Lisbonne, Portugal, $<$ halshs-01123906>.

Epstein-Pliouchtch M. et Fuchs R. (2008). « Myth, History and Conservation in Tel Aviv », D. Van Den Heuvel et M. Mesman (dir.), The Challenge of change, Dealing with the legacy of the modern Movement, Amsterdam, Ios Press et Delft University Press, p. 109114.

EPSTEIN-PliouchtCh M. (2012). «Un portrait narratif de la modernité israélienne : l'urbain et le rural », in M. CASCIATO et E. D'ORGEIX, Architectures modernes. L'émergence d'un patrimoine, Wavre, Mardaga, p. 51-60.

Ferretti F. (2012). «Aux origines de l'aménagement régional : le schéma de la Valley Section de Patrick Geddes (1925)», M@ppemonde, vol. 108, n²4, p. 1-13.

FISHER Y. (1984). Tel Aviv : 75 years of art, Massada, Ramat-Gan, 207 p.

FREY J.-P. (2011). «Un objet de controverse : la monumentalité entre architecture et forme urbaine », in M. GRAVARI-BARBAS et I. IOSA, Monumentalité(s) urbaine(s) aux XIXe et XXe siècles. Sens, formes et enjeux urbains, Paris, L'Harmattan, p. 207-226.

Helman A. (2002). "'Even the Dogs in the Street Bark in Hebrew' : National Ideology and Everyday Culture in Tel Aviv», The Jewish Quarterly Review, vol. 92, n³-4, p. 359-382.

Girard P. et Fayolle Lussac B. (coord.) (1996). Cités, Cités-Jardins : une histoire européenne. Talence : Editions de la MSH d'Aquitaine, 262 p.

Gravari-BARBAS M. (2012). «Tourisme et patrimoine, le temps des synergies ?», in C. KHAZNADAR (dir.), Le patrimoine oui, mais quel patrimoine?, Paris, Éditions Babel, p. 375399.

Gravari-Barbas M. et Renard-Delautre C. (2015). Starchitecture(s). Figures d'archictes et espace urbain, Condé-sur-Noireau, L'Harmattan, 270 p.

Herzl T. (2004). Altneuland. Nouveau pays ancien (1 ${ }^{\text {ère }}$ édition 1902), Cahors, Éditions de l'Éclat, 361 p.

Hyman B. (1994). British planners in Palestine, 1918-1936, Th. de Doctorat, London School of Economics, Université de Londres, 781 p.

Hoffmann J. (2016). « La ville adaptative: la vision de Geddes dans l'évolution de Tel-Aviv (1948-2003) », Espaces et Sociétés, n¹67, vol. 4, p. 63-79. 
Home R. (2013). On Planting and Planning : the Making of British Colonial City, Londres, Routledge, 272 p. (édition originale 1996).

Howard E. (1998). Cités-jardins de demain, Paris, Sens et Tonka ( $1^{\text {ère }}$ édition anglaise, 1902), $216 \mathrm{p}$.

Hisler-Rubin N. (2011). Patrick Geddes and Town Planning. A critical view. Londres - New York, Routledge, 205 p.

Ingersoll R. (1995). « Pilgrimage to the White City : International Style Conference at TelAviv », Journal of Architectural Education, vol. 48, n4, p. 268-270.

JoKILHETO J. (2006). «Modern built heritage as World Heritage», in Journal of the International Day for Monuments and Sites, pp. 45- 47

KhaZnADAR C. (2012). Le patrimoine, oui, mais quel patrimoine? Arles, Actes Sud, coll. Babel, 482 p.

KATZ Y. (1986). «Ideology and urban development: Zionism and the origins of Tel-Aviv, 1906-1914 », Journal of Historical Geography, n²12, vol. 4, p. 402-424.

KIPNIS B. (2004). «Tel Aviv, Israel - A World City in Evolution : Urban Development at a Deadend of the Global Economy », Dela, n²1, p. 183-193.

LE Goff J. (dir.) (1997). Patrimoine et passions identitaires, Paris, Fayard, 445 p.

LENIAUD J-M. (2001). Chroniques patrimoniales, Bruxelles, Norma, 494 p.

LEvin M. (1984). White City International Style Architecture in Israel, Portrait of an Era, Tel-Aviv, The Tel Aviv Museum, 72 p.

LEVINE M. (2005). Overthrowing geography: Jaffa, Tel Aviv, and the struggle for Palestine, 1880-1948, Berkeley et Los Angeles, University of California Press, 442 p.

Machat Ch., Petzet M. et ZIESEMER J. (éd.) (2010). Heritage at risk : Icomos World Report 2008-2010 on Monuments and Sites in Danger, Berlin, Hendrik Bäßler Verlag, 212 p.

Mann B. (2006). A place in History. Modernism, Tel Aviv, and the creation of the Jewish Urban Space, Stanford, Stanford University Press, 336 p.

Melot M. (2012). Mirabilia. Essai sur l'Inventaire général du patrimoine culturel. Paris, Gallimard, coll. $N R F, 286$ p. 
Metzger-Szmuk N. (2004). Des maisons sur le sable. Tel-Aviv: mouvement moderne et esprit Bauhaus, Éditions de l'éclat, Paris et Tel-Aviv, 447 p.

Mualam N. et SybBlis M. (2016). " The functional threshold of modern heritage : form versus function and the struggle over Tel Aviv's concert hall », International Journal of Heritage Studies, 22 :2, pp. 145-164.

Tournikiotis P. (2012). « La liste et le type. De l'inventaire thématique à la (ré)écriture de l'histoire de l'architecture moderne », M. CASCiato et E. D'Orgeix (dir.), Architectures modernes. Emergence d'un patrimoine, Wavre, Mardaga, p. 19-26.

PAyton N. (1995). "The machine in the garden city: Patrick Geddes' plan for Tel Aviv », Planning Perspectives vol. 10, n4, p. 359-381.

Ram U (2008) The Globalization of Israel. McWolrd in Tel Aviv, Jihad in Jerusalem, Londres - New York, Routledge, 290 p.

Rautenberg M. (2015). «L'urbanité comme patrimoine de la ville », Diogène, n'151-152, vol. 3-4, pp. 90-102.

RIEGL A. (2003). Le culte moderne des monuments, Paris, L'Harmattan, 124 p. (édition originale : 1903).

Rofe Y. (2009). «The White City of Tel-Aviv », Planum, URL: http://www.planum.net/thewhite-city-of-tel-aviv-1.

Roger A. (1997). Court traité du Paysage, Paris, Gallimard, coll. NRF, 216 p.

Rotbard S. (2015). White City, Black City: Architecture and War in Tel-aviv and Jaffa, Londres, Pluto Press, 244 p.

RozenholC C. (2009). "Tel-Aviv a cent ans ! 1909-2009: un siècle de globalisation au Proche-Orient », Echogéo, n8, p. 1-8, http://echogeo.revues.org/10955.

RozenHOlC C. (2014). "Sens du lieu, identifications et méditerranéité à Tel-Aviv et Marseille. Une proposition méthodologique pour l'analyse de la mondialisation des lieux en Méditerranée », in Géographie et cultures, n89-90, p. 261-282.

RozenholC C. (2018). Tel-Aviv. Le quartier de Florentine : un ailleurs dans la ville, Paris, Créaphis, $237 \mathrm{p}$.

RozenholC C. et Fijalkow Y. (2016). «D'une tente à l'autre. 'Crise du logement' et mobilisations sociales en France et en Israël », Annales de géographie, n707, 2016, p. 1-19. 
SHARon A. (1976). Kibbutz + Bauhaus an architect's way a new land, Massada Publishing Ltd, $260 \mathrm{p}$.

SchlÖR J. (1999). Tel Aviv. From Dream to City, London, Reaktion Books, 1999, 339 p.

Tufano A. (2016). "Man's Role in Changing the Face of the Earth (1955): From the Rediscovery of Marsh to the Emergence of Urban Metabolism », Global Environment, vol. $10, \mathrm{n}^{\circ} 2$, pp. 335-362.

TURNER J. (1984). White City International Style Architecture in Israel. Judith Turner: Photographs, Tel-Aviv, The Tel Aviv Museum, 88 p.

Tzfadia E. et Yacobi H. (2011). Rethinking Israeli Space. Periphery and Identity, Oxon et New York, Routledge, 153 p.

WALter F. (2004). Les figures paysagères de la nation. Territoire et paysage en Europe (16e-20e siècle), Paris, Éditions de l'EHESS, 524 p.

Welter V. (2002). Biopolis. Patrick Geddes and the City of Life, Cambridge, MA, The MIT Press, 379 p.

Welter V. (2009). « The 1925 Master Plan for Tel-Aviv by Patrick Geddes », Israel Studies, vol. $14, \mathrm{n}^{\circ} 3$, p. 94-119.

Weill-Rochant C. (2006). Le plan de Patrick Geddes pour la Ville Blanche de Tel Aviv: une part d'ombre et de lumière, Thèse de Doctorat, Université Paris VIII, 670 p.

WeILl-Rochant C. (2008). L'atlas de Tel-Aviv. 1908-2008, Paris, Éditions du CNRS, 160 p.

WeILl-Rochant C. (2008). «Évaluer la pérennité urbaine. L'exemple du plan Geddes pour Tel Aviv », C. Vallat (dir.) Pérennité urbaine. La ville par de-là ses métamorphoses, vol. 1 Traces, Paris, L’Harmattan, p. 314-330.

WeIZMAN E. (2007), Hollow Land. Israel's architecture of occupation, Londres et New York, Verso, 318 p. 